\title{
THE ASYMPTOTIC NORMALITY OF AN ADJUSTED LEAST SQUARES ESTIMATOR IN A MULTIVARIATE VECTOR ERRORS-IN-VARIABLES REGRESSION MODEL
}

UDC 519.21

\author{
I. O. SEN'KO
}

\begin{abstract}
An adjusted least squares estimator in a linear multivariate vector errorin-variables regression model is considered in this paper. Conditions for the asymptotic normality of this estimator are given. A modification of the estimator is constructed whose asymptotic properties are the same as those of the adjusted least squares estimator and which is stable even if a sample is small.
\end{abstract}

\section{INTRODUCTION}

Errors-in-variables models are an important generalization of linear regression models, since there are a number of applied problems for which the data are obtained as a result of a sampling experiment and should include some errors. Those models appear, in particular, in problems of identification of dynamic systems, in signal processing as well as in geological and oceanographic studies. A survey of errors-in-variables models can be found in 3. An estimation of parameters in those models should necessarily account for errors in both dependent and independent variables.

In this paper, we consider a linear errors-in-variables model described by an overdefined system of linear equations. The model can be written in the matrix form as

$$
A X=B,
$$

where the matrices $A$ and $B$ are observed with errors and where $X$ is an unknown matrix to be estimated. The covariance matrix of errors for variables in matrix $A$ is assumed to be known, while that for matrix $B$ is, in contrast, unknown. We consider an adjusted least squares estimator of the matrix parameter $X$. Sufficient conditions for the consistence and strong consistence for this model are obtained in [7]. Some further references for other estimators in linear errors-in-variables models can also be found in [7].

In this paper, we establish conditions for the asymptotic normality of the estimator for the case where the number of rows in the matrix $A$ increases. A modification of the estimator is constructed with the property that the modification is more stable from the computational point of view and has the same asymptotic behavior. The idea of the modification is first used in [2] for a scalar polynomial errors-in-variables model.

This paper is organized as follows. The model of observations is described in Section 2 . Some preliminary results are also stated in Section 2, The asymptotic normality is proved, a consistent estimator of the asymptotic covariance matrix is established, and the confidence ellipsoid for the matrix $X$ is constructed in Section 3 . A modification

2010 Mathematics Subject Classification. Primary 62J12.

Key words and phrases. Error-in-variables models, adjusted least squares estimator, asymptotic normality, small samples. 
of the estimator for small samples is considered in Section 4 Concluding remarks are included in Section 5. The proofs are collected in the Appendix.

The following notation is used throughout the paper. We assume that all vectors are written as columns; $\|z\|$ is the Euclidean norm of a vector $z \in \mathbb{R}^{n} ; I_{n}$ is the unit $n \times n$ matrix; for a matrix $Z \in \mathbb{R}^{m \times n}, Z=\left(z_{i j}\right)_{i=1, j=1}^{n}$, let $\|Z\|$ denote the operator norm corresponding to the Euclidean norms in $\mathbb{R}^{n}$ and $\mathbb{R}^{m}$; further let

$$
\|Z\|_{\mathrm{F}}=\sqrt{\sum_{i=1}^{m} \sum_{j=1}^{n} z_{i j}^{2}}
$$

be the Frobenius norm of a matrix $Z$. By $Z^{\dagger}$, we denote the pseudo-inverse matrix 6 , for $Z$. The expectation and variance are denoted by symbols $E$ and Var, respectively. If $\left\{X_{m} \in \mathbb{R}^{n \times d}, m \geqslant 0\right\}$ is a sequence of random matrices, then the notation $X_{m} \stackrel{\mathrm{P}}{\rightarrow} X_{0}$, $m \rightarrow \infty$ means that $\left\|X_{m}-X_{0}\right\| \stackrel{\mathrm{P}}{\rightarrow} 0, m \rightarrow \infty$. The almost sure convergence $X_{m} \stackrel{\mathrm{P} 1}{\longrightarrow} X_{0}$, $m \rightarrow \infty$ of random matrices is defined similarly.

The notation $X_{m}=\mathrm{op}(1), m \rightarrow \infty$ means that $X_{m} \stackrel{\mathrm{P}}{\rightarrow} 0, m \rightarrow \infty$, while $X_{m}=O_{\mathrm{P}}(1)$, $m \rightarrow \infty$ means that a sequence of random variables $\left\{\left\|X_{m}\right\|\right\}$ is stochastically bounded. For a square symmetric matrix $V$, we denote by $\lambda_{\min }(V)$ and $\lambda_{\max }(V)$ its minimal and maximal eigenvalue, respectively. The notation $U>V$ for two square matrices $U$ and $V$ of the same size means that the matrix $U-V$ is positive definite, while $U \geqslant V$ means that the matrix $U-V$ is nonnegative definite. We write

$$
\operatorname{vec}(W)=\left[\begin{array}{llll}
w_{1}^{T} & w_{2}^{T} & \cdots & w_{d}^{T}
\end{array}\right]^{T} \in \mathbb{R}^{q d}
$$

for a rectangular matrix $W=\left[\begin{array}{llll}w_{1} & w_{2} & \ldots & w_{d}\end{array}\right] \in \mathbb{R}^{q \times d}$. Let

$$
Z \otimes W=\left[\begin{array}{cccc}
z_{11} W & z_{12} W & \ldots & z_{1 n} W \\
z_{21} W & z_{22} W & \ldots & z_{2 n} W \\
\ldots & & & \\
z_{m 1} W & z_{m 2} W & \ldots & z_{m n} W
\end{array}\right]
$$

denote the Kronecker product of rectangular matrices $Z$ and $W$. Finally, the symbol const denotes an arbitrary constant that does not depend on the number of observations $m$.

\section{THE MODEL OF OBSERVATIONS}

Let $\mathfrak{X}$ be an unknown linear operator acting from $\mathbb{R}^{n}$ to $\mathbb{R}^{d}, \mathfrak{X} z=X_{0}^{T} z, z \in \mathbb{R}^{n}$. Here $X_{0}=\left(x_{i j}^{0}\right)_{i=1, j=1}^{n}$. Some sets of input and output data are observed and used to estimate $X$; assume that the observations contain random errors. By $a_{i}^{0} \in \mathbb{R}^{n}, i \geqslant 1$, we denote nonrandom vectors, corresponding to the true values of the input data; similarly, let $b_{i}^{0} \in \mathbb{R}^{d}, i \geqslant 1$ be the vectors corresponding to the true values of the output data; the coordinates of these vectors are denoted by $a_{i j}^{0}$ and $b_{i j}^{0}$, respectively.

Assume that the true vectors are such that

$$
b_{i}^{0}=X_{0}^{T} a_{i}^{0}, \quad i \geqslant 1 .
$$

Further let one observe vectors $a_{i} \in \mathbb{R}^{n}$ and $b_{i} \in \mathbb{R}^{d}, 1 \leqslant i \leqslant m$, and let

$$
a_{i}=a_{i}^{0}+\tilde{a}_{i}, \quad b_{i}=b_{i}^{0}+\tilde{b}_{i},
$$

where $\tilde{a}_{i}$ and $\tilde{b}_{i}$ are centered random errors of observations. The coordinates of these vectors are denoted by $a_{i j}, \tilde{a}_{i j}, b_{i j}$, and $\tilde{b}_{i j}$. Then the linear vector model with errorsin-variables is given by

$$
b_{i}=X_{0}^{T} a_{i}^{0}+\tilde{b}_{i}, \quad a_{i}=a_{i}^{0}+\tilde{a}_{i}, \quad 1 \leqslant i \leqslant m .
$$


Using the notation $A=\left[\begin{array}{llll}a_{1} & a_{2} & \ldots & a_{m}\end{array}\right]^{T}=\left(a_{i j}\right)_{i=1, j=1}^{m}$ and similar notation for $A_{0}$, $\tilde{A}, B, B_{0}, \tilde{B}$, where $A=A_{0}+\tilde{A}, B=B_{0}+\tilde{B}$, the latter model can be rewritten as an approximate equality

$$
A X_{0} \approx B
$$

under the condition that

$$
A_{0} X_{0}=B_{0} \text {. }
$$

The covariance matrix of errors in the regressor is denoted by $V_{\tilde{A}}=\mathrm{E} \tilde{A}^{T} \tilde{A}$ and is supposed to be known.

Below is the main assumption concerning the observations.

(i) : The errors $\left\{\tilde{a}_{i j}, i \geqslant 1,1 \leqslant j \leqslant n\right\}$ and $\left\{\tilde{b}_{i l}, i \geqslant 1,1 \leqslant l \leqslant d\right\}$ are jointly independent, centered, and possess finite second moments.

Following the so-called corrected score method [1, Chapter 7], one can construct an adjusted least squares estimator of the matrix parameter $X_{0}$,

$$
\hat{X}=\left(A^{T} A-V_{\tilde{A}}\right)^{\dagger} A^{T} B .
$$

Put $V_{A_{0}}=A_{0}^{T} A_{0}$. Conditions for the consistence and strict consistence of this estimator are found in [7] (see below).

Theorem 2.1 (Consistence). Let assumption (ii) hold. Assume further that

(ii) : the rows of the matrix $\tilde{A}$ are independent (that is, the vectors $\left\{\tilde{a}_{i}, i \geqslant 1\right\}$ are independent) and the rows of matrix $\tilde{B}$ are independent (in other words, the vectors $\left\{\tilde{b}_{i}, i \geqslant 1\right\}$ are independent).

(iii) : $\mathrm{E} \tilde{a}_{i j}^{4} \leqslant$ const; $\mathrm{E} \tilde{b}_{i l}^{2} \leqslant$ const.

(iv) :

$$
\frac{\lambda_{\max }\left(V_{A_{0}}\right)+m}{\lambda_{\min }^{2}\left(V_{A_{0}}\right)} \rightarrow 0, \quad m \rightarrow \infty
$$

Then $\hat{X} \stackrel{\mathrm{P}}{\rightarrow} X_{0}, m \rightarrow \infty$.

Theorem 2.2 (Strict consistence). Let assumptions (ii) and (iii) hold. We further assume that

$(\mathbf{v})$ : there exists a number $r \geqslant 2$ such that $\mathrm{E}\left|\tilde{a}_{i j}\right|^{2 r} \leqslant$ const and $\mathrm{E}\left|\tilde{b}_{i j}\right|^{2 r} \leqslant$ const.

(vi) : The number $r$ in condition (四) is such that

$$
\sum_{m=m_{0}}^{\infty}\left(\frac{m^{r / 2}}{\lambda_{\min }^{r}\left(V_{A_{0}}\right)}+\frac{\lambda_{\max }^{r}\left(V_{A_{0}}\right)}{\lambda_{\min }^{2 r}\left(V_{A_{0}}\right)}\right)<\infty
$$

for some $m_{0} \geqslant 1$.

Then

$$
\hat{X} \stackrel{\mathrm{P} 1}{\longrightarrow} X_{0}, \quad m \rightarrow \infty .
$$

\section{Asymptotic nORMALITY}

3.1. A theorem on the asymptotic normality. The proof of the asymptotic normality uses assumptions (ii)-(iii) together with the following additional assumptions.

(vii) : $\frac{1}{m} V_{A_{0}} \rightarrow V_{A_{\infty}}, m \rightarrow \infty$, where $V_{A_{\infty}}>0$.

The latter condition implies that

$$
m^{-1} \lambda_{\max }\left(V_{A_{0}}\right) \rightarrow \lambda_{\max }\left(V_{A_{0}}\right), \quad m \rightarrow \infty,
$$

and

$$
m^{-1} \lambda_{\min }\left(V_{A_{0}}\right) \rightarrow \lambda_{\min }\left(V_{A_{0}}\right), \quad m \rightarrow \infty .
$$

Note that condition (iv) follows from (vii). 
(viii) : The rows of the matrix $\tilde{A}$ are identically distributed and the rows of the matrix $\tilde{B}$ are identically distributed; in other words, the vectors $\tilde{a}_{i} \sim \tilde{a}, i \geqslant 1$ are identically distributed and the vectors $\tilde{b}_{i} \sim \tilde{b}, i \geqslant 1$ are identically distributed.

Let

$$
V_{\tilde{a}}=\operatorname{Cov}\left(\tilde{a}_{i}\right)=\frac{1}{m} V_{\tilde{A}}, \quad V_{\tilde{b}}=\operatorname{Cov}\left(\tilde{b}_{i}\right)=\frac{1}{m} \mathrm{E} \tilde{B}^{T} \tilde{B} .
$$

To distinguish between the vectors $\tilde{a}_{i}$ and coordinates of the vector $\tilde{a}$, the latter are denoted by $\tilde{a}(i), i=1, \ldots, n$. Similarly, the coordinates of the vector $\tilde{b}$ are denoted by $\tilde{b}(i), i=1, \ldots, d$.

Further we introduce some additional assumptions:

(ix) : there exists a number $\delta>0$ such that $\mathrm{E}|\tilde{a}(j)|^{4+\delta}<\infty, j=1, \ldots, n$;

$(\mathbf{x})$ : there exists a number $\tau>0$ such that $\sup _{m \geqslant 1} \frac{1}{m} \sum_{i=1}^{m}\left\|a_{i}^{0}\right\|^{2+\tau}<\infty$;

(xi) : the $\operatorname{limit}_{m \rightarrow \infty} \frac{1}{m} \sum_{i=1}^{m} a_{i}^{0} \stackrel{\text { def }}{=} a^{0}$ exists.

The coordinates of the vector $a^{0}$ are denoted by $a^{0}(i), i=1, \ldots, n$.

Finally, assumption (xii) written below is needed to prove that the asymptotic covariance matrix is positive definite.

$$
\text { (xii) : } V_{\tilde{a}}>0 \text {. }
$$

Theorem 3.1 (Asymptotic normality). 1) Let assumptions (ii)-(iii) and (vii)-(xi) hold. Then

$$
\sqrt{m} \cdot \operatorname{vec}\left(\hat{X}-X_{0}\right) \stackrel{d}{\rightarrow} N(0, \Sigma), \quad m \rightarrow \infty,
$$

where the matrix $\Sigma$ is defined by equalities (6) and (11).

2) If additionally assumption (xii) holds, then $\Sigma>0$.

Proof. 1) Assumption (viil) implies that there exists a number $m_{0} \in \mathbb{N}$ such that

$$
\operatorname{det} V_{A_{0}} \neq 0
$$

for all $m \geqslant m_{0}$. Then equality (11) can be rewritten for $m \geqslant m_{0}$ as follows

$$
\left(A^{T} A-V_{\tilde{A}}\right) \hat{X}=A^{T}\left(B_{0}+\tilde{B}\right)
$$

or

$$
V_{A_{0}}^{-1}\left(A^{T} A-V_{\tilde{A}}\right) \hat{X}=V_{A_{0}}^{-1}\left(A^{T} A_{0} X_{0}+A^{T} \tilde{B}\right) .
$$

Consider the difference $\hat{X}-X_{0}$ on the left-hand side:

$$
V_{A_{0}}^{-1}\left(A^{T} A-V_{\tilde{A}}\right)\left(\hat{X}-X_{0}\right)=V_{A_{0}}^{-1} A^{T} \tilde{B}+V_{A_{0}}^{-1}\left(A^{T} A_{0} X_{0}-\left(A^{T} A-V_{\tilde{A}}\right) X_{0}\right) \text {. }
$$

Assumptions (ii) - (iii) and (vii) imply the weak consistence in Theorem 2.1, The proof of this result in [7] is based on the relation

$$
V_{A_{0}}^{-1}\left(A^{T} A-V_{\tilde{A}}\right)=I_{n}+o_{\mathrm{P}}(1) .
$$

Then (2) is rewritten as follows

$$
\left(I_{n}+o_{\mathrm{P}}(1)\right)\left(\hat{X}-X_{0}\right)=V_{A_{0}}^{-1}\left(A^{T} \tilde{B}+V_{\tilde{A}} X_{0}-A^{T} \tilde{A} X_{0}\right) .
$$

Let

$$
l=\operatorname{vec}\left(A^{T} \tilde{B}+V_{\tilde{A}} X_{0}-A^{T} \tilde{A} X_{0}\right)=\operatorname{vec}\left(\sum_{i=1}^{m} a_{i} \tilde{b}_{i}^{T}+\sum_{i=1}^{m} \mathrm{E} \tilde{a}_{i} \tilde{a}_{i}^{T} X_{0}-\sum_{i=1}^{m} a_{i} \tilde{a}_{i}^{T} X_{0}\right) .
$$

As shown in the Appendix,

$$
\frac{l}{\sqrt{m}} \stackrel{d}{\rightarrow} N\left(0, \Sigma_{1}\right), \quad m \rightarrow \infty
$$

where $\Sigma_{1}$ is defined by (11). 
Thus

$$
\sqrt{m}\left(I_{n}+o_{\mathrm{P}}(1)\right) \operatorname{vec}\left(\hat{X}-X_{0}\right)=\left(I_{d} \otimes\left(\frac{V_{A_{0}}}{m}\right)^{-1}\right) \frac{l}{\sqrt{m}},
$$

whence

$$
\sqrt{m} \operatorname{vec}\left(\hat{X}-X_{0}\right) \stackrel{d}{\rightarrow}\left(I_{d} \otimes V_{A_{\infty}}^{-1}\right) N\left(0, \Sigma_{1}\right)=N(0, \Sigma),
$$

where

$$
\Sigma=\left(I_{d} \otimes V_{A_{\infty}}^{-1}\right) \Sigma_{1}\left(I_{d} \otimes V_{A_{\infty}}^{-1}\right)^{T}
$$

is the asymptotic covariance matrix.

2) The proof is given in the Appendix.

\section{Corollary 3.1.}

$$
\begin{aligned}
\left\|\sqrt{m} \Sigma^{-1 / 2} \operatorname{vec}\left(\hat{X}-X_{0}\right)\right\|^{2} & =m \cdot \operatorname{vec}^{T}\left(\hat{X}-X_{0}\right) \Sigma^{-1} \operatorname{vec}\left(\hat{X}-X_{0}\right) \\
& \stackrel{d}{\rightarrow} \chi_{n d}^{2}, \quad m \rightarrow \infty .
\end{aligned}
$$

3.2. Confidence ellipsoid for $X_{0}$. In order to construct a confidence ellipsoid, one needs to consider a consistent estimator of the matrix $\Sigma$. The following assumption allows one to construct such an estimator.

(xiii) : The third and fourth moments of the vector of errors for hidden variables are known, that is, the following sets of numbers

$$
\{\mathrm{E} \tilde{a}(i) \tilde{a}(j) \tilde{a}(k) \mid i, j, k=1, \ldots, d\}
$$

and

$$
\{\mathrm{E} \tilde{a}(i) \tilde{a}(j) \tilde{a}(k) \tilde{a}(l) \mid i, j, k, l=1, \ldots, d\}
$$

are known.

The moments involved in assumption (xiii) can be estimated, for example, if the observations are repeated.

Assumption (xiii) implies that the term $\mathrm{E}(\tilde{a} \otimes \tilde{a})(\tilde{a} \otimes \tilde{a})$ in expression (11) is known and that the factor $a^{0}(j)$ in the entry $a^{0}(j) \mathrm{E} \tilde{a}(i) \tilde{a}(k) \tilde{a}(l)$ of the matrix

$$
\mathrm{E}\left[\left(\tilde{a} \otimes a^{0}\right)(\tilde{a} \otimes \tilde{a})\right]
$$

can be estimated by

$$
\widehat{a^{0}(j)}=\frac{1}{m} \sum_{p=1}^{m} a_{p j}
$$

Moreover, the latter estimator is consistent, $\widehat{a^{0}(j)} \stackrel{\mathrm{P}}{\rightarrow} a^{0}(j), m \rightarrow \infty$.

Denote by $U_{A \text {,app }}$ the consistent estimator of the matrix $\mathrm{E}\left[\left(\tilde{a} \otimes a^{0}\right)(\tilde{a} \otimes \tilde{a})\right]$. In other words, this estimator is such that

$$
U_{A, \text { app }} \underset{m \rightarrow \infty}{\stackrel{\mathrm{P}}{\longrightarrow}} \mathrm{E}\left[\left(\tilde{a} \otimes a^{0}\right)(\tilde{a} \otimes \tilde{a})\right] .
$$

Our current aim is to construct a consistent estimator for the matrix $V_{A_{\infty}}$. Using equality (3) we get

$$
\begin{aligned}
& \left(\frac{1}{m} A_{0}^{T} A_{0}\right)^{-1}\left(\frac{1}{m} A^{T} A-V_{\tilde{a}}\right) \stackrel{\mathrm{P}}{\rightarrow} I_{n}, \quad m \rightarrow \infty, \\
& \hat{V}_{A_{\infty}} \stackrel{\text { def }}{=} \frac{1}{m} A^{T} A-V_{\tilde{a}} \stackrel{\mathrm{P}}{\rightarrow} V_{A_{\infty}} .
\end{aligned}
$$


To estimate the covariance matrix $V_{\tilde{b}}$, we use the equality $B=A_{0} X_{0}+\tilde{B}$. Then

$$
\begin{gathered}
\mathrm{E} B^{T} B=X_{0}^{T}\left(A_{0}^{T} A_{0}\right) X_{0}+\mathrm{E} \tilde{B}^{T} \tilde{B}, \\
\frac{1}{m} \mathrm{E} B^{T} B=X_{0}^{T}\left(\frac{1}{m} A_{0}^{T} A_{0}\right) X_{0}+V_{\tilde{b}} .
\end{gathered}
$$

Since $\frac{1}{m} \mathrm{E} B^{T} B=\frac{1}{m} \sum_{i=1}^{m} \mathrm{E} b_{i} b_{i}^{T}$, we are in a position to prove the law of large numbers for the sequence of matrices $\left\{b_{i} b_{i}^{T}, i \geqslant 1\right\}$, namely

$$
\frac{1}{m} \sum_{i=1}^{m} b_{i} b_{i}^{T}-\frac{1}{m} \sum_{i=1}^{m} \mathrm{E} b_{i} b_{i}^{T} \stackrel{\mathrm{P}}{\rightarrow} 0, \quad m \rightarrow \infty .
$$

We have

$$
\frac{1}{m} \sum_{i=1}^{m} b_{i} b_{i}^{T}=\frac{1}{m} \sum_{i=1}^{m} b_{i}^{0} b_{i}^{0 T}+\frac{1}{m} \sum_{i=1}^{m} b_{i}^{0} \tilde{b}_{i}^{T}+\frac{1}{m} \sum_{i=1}^{m} \tilde{b}_{i} b_{i}^{0 T}+\frac{1}{m} \sum_{i=1}^{m} \tilde{b}_{i} \tilde{b}_{i}^{T} .
$$

Then we are going to show that

$$
\frac{1}{m} \sum_{i=1}^{m} b_{i}^{0} \tilde{b}_{i}^{T} \rightarrow \mathrm{E}\left(\frac{1}{m} \sum_{i=1}^{m} b_{i}^{0} \tilde{b}_{i}^{T}\right)=0, \quad m \rightarrow \infty .
$$

With this goal in mind, we apply the Chebyshev law of large numbers to the sequence of independent random variables $\left\{b_{i j}^{0} \tilde{b}_{i k}, i \geqslant 1\right\}, j, k=1, \ldots, n$. We need to show that

$$
\operatorname{Var}\left(b_{i j}^{0} \tilde{b}_{i k}\right)=o(i), \quad i \rightarrow \infty
$$

and

$$
\begin{aligned}
\operatorname{Var}\left(b_{i j}^{0} \tilde{b}_{i k}\right) & =\left(b_{i j}^{0}\right)^{2} \mathrm{E}\left(\tilde{b}_{i k}\right)^{2} \leqslant\left\|b_{i}^{0}\right\|^{2} \cdot \text { const }=\text { const } \cdot\left\|X_{0} a_{i}^{0}\right\|^{2} \\
& \leqslant \text { const } \cdot\left\|X_{0}\right\|^{2}\left\|a_{i}^{0}\right\|^{2} .
\end{aligned}
$$

Next, since $\frac{1}{m} \sum_{i=1}^{m} a_{i}^{0} a_{i}^{0 T} \rightarrow V_{\infty}, m \rightarrow \infty$, we get

$$
\begin{gathered}
\frac{1}{m} a_{m}^{0} a_{m}^{0 T}=\frac{1}{m} \sum_{i=1}^{m} a_{i}^{0} a_{i}^{0 T}-\frac{m-1}{m} \cdot \frac{1}{m-1} \sum_{i=1}^{m-1} a_{i}^{0} a_{i}^{0 T} \underset{m \rightarrow \infty}{\longrightarrow} V_{A_{\infty}}-1 \cdot V_{A_{\infty}}=0, \\
\left\|a_{i}^{0} a_{i}^{0 T}\right\|_{\mathrm{F}}=\left(\sum_{j=1, k=1}^{m}\left(a_{i j}^{0} a_{i k}^{0}\right)^{2}\right)^{1 / 2}=\left(\sum_{j=1}^{m}\left(a_{i j}^{0}\right)^{2} \sum_{k=1}^{m}\left(a_{i k}^{0}\right)^{2}\right)^{1 / 2}=\left\|a_{i}\right\|^{2} .
\end{gathered}
$$

Thus $\left\|a_{i}\right\|^{2}=o(i), i \rightarrow \infty$, whence

$$
\operatorname{Var}\left(b_{i j}^{0} \tilde{b}_{i k}\right) \leqslant \text { const } \cdot\left\|a_{i}\right\|^{2}=o(i), \quad i \rightarrow \infty .
$$

Then

$$
\frac{1}{m} \sum_{i=1}^{m} b_{i}^{0 T} \tilde{b}_{i}=\left(\frac{1}{m} \sum_{i=1}^{m} b_{i}^{0} \tilde{b}_{i}^{T}\right)^{T} \rightarrow 0, \quad m \rightarrow \infty .
$$

Further, taking into account assumptions (iii) and (viii), we apply the strong law of large numbers (Theorem 3 in [9, Section IV.3]) to each entry of the sequence of the matrices $\left\{\tilde{b}_{i} \tilde{b}_{i}^{T}, i \geqslant 1\right\}$. As a result we conclude that

$$
\frac{1}{m} \sum_{i=1}^{m} \tilde{b}_{i} \tilde{b}_{i}^{T} \stackrel{\mathrm{P} 1}{\longrightarrow} \operatorname{Cov}(\tilde{b})=V_{\tilde{b}}, \quad m \rightarrow \infty .
$$


Therefore,

$$
\begin{aligned}
\frac{1}{m} \sum_{i=1}^{m} b_{i} b_{i}^{T}-\frac{1}{m} \sum_{i=1}^{m} \mathrm{E} b_{i} b_{i}^{T}= & \frac{1}{m} \sum_{i=1}^{m} b_{i}^{0} \tilde{b}_{i}^{T}+\frac{1}{m} \sum_{i=1}^{m} \tilde{b}_{i} b_{i}^{0 T}+\frac{1}{m} \sum_{i=1}^{m} \tilde{b}_{i} \tilde{b}_{i}^{T}-V_{\tilde{b}} \\
& \underset{m \rightarrow \infty}{\longrightarrow} 0+0+V_{\tilde{b}}-V_{\tilde{b}}=0 .
\end{aligned}
$$

This means that

$$
\hat{V}_{\tilde{b}} \stackrel{\text { def }}{=} \frac{1}{m} \sum_{i=1}^{m} b_{i}^{T} b_{i}-\hat{X}^{T}\left(\frac{1}{m} A^{T} A-V_{\tilde{a}}\right) \hat{X} \stackrel{\mathrm{P}}{\rightarrow} V_{\tilde{b}}, \quad m \rightarrow \infty,
$$

is a consistent estimator for $V_{\tilde{b}}$. Finally,

$$
\begin{aligned}
& \hat{\Sigma}_{1} \stackrel{\text { def }}{=} \hat{V}_{\tilde{b}} \otimes \hat{V}_{A_{\infty}}+\hat{V}_{\tilde{b}} \otimes V_{\tilde{a}} \\
&+\left(\hat{X} \otimes I_{n}\right) \\
& \quad \times\left[V_{\tilde{a}} \otimes \hat{V}_{A_{\infty}}+\mathrm{E}(\tilde{a} \otimes \tilde{a})(\tilde{a} \otimes \tilde{a})-\operatorname{vec}\left(V_{\tilde{a}}\right) \operatorname{vec}^{T}\left(V_{\tilde{a}}\right)+U_{A, \text { app }}+U_{A, \text { app }}^{T}\right] \\
& \times\left(\hat{X} \otimes I_{n}\right)^{T} \stackrel{\mathrm{P}}{\rightarrow} \Sigma_{1}, \quad m \rightarrow \infty,
\end{aligned}
$$

and

$$
\hat{\Sigma} \stackrel{\text { def }}{=}\left(I_{d} \otimes \hat{V}_{A_{\infty}}^{-1}\right) \hat{\Sigma}_{1}\left(I_{d} \otimes \hat{V}_{A_{\infty}}^{-1}\right)^{T} \stackrel{\mathrm{P}}{\rightarrow} \Sigma, \quad m \rightarrow \infty .
$$

Considering relation (7), the confidence ellipsoid for the vector parameter vec $\left(X_{0}\right)$ corresponding to the confidence probability $\gamma$ can be described in the space $\mathbb{R}^{\text {nd }}$ of the coordinates $\left(x_{11}, x_{21}, \ldots, x_{n 1}, x_{12}, x_{22}, \ldots, x_{n 2}, \ldots, x_{1 d}, x_{2 d}, \ldots, x_{n d}\right)$ by the following system of inequalities

$$
(\operatorname{vec}(X)-\operatorname{vec}(\hat{X}))^{T}\left(\frac{1}{m} \hat{\Sigma}\right)^{-1}(\operatorname{vec}(X)-\operatorname{vec}(\hat{X})) \leqslant \chi_{n d, \gamma}^{2}
$$

where $X=\left(x_{i j}\right), i=1, \ldots, n, j=1, \ldots, d$ and $\chi_{n d, \gamma}^{2}$ is a quantile of order $\gamma$ for the $\chi^{2}$ distribution with $n d$ degrees of freedom, that is, $\mathrm{P}\left(\chi_{n d}^{2} \leqslant \chi_{n d, \gamma}^{2}\right)=\gamma$.

\section{A MODificAtion FOR SMALL SAMPLES}

There is a problem with using the above estimator for numerical calculations. The problem is related to the fact that the matrix $\left(A^{T} A-V_{\tilde{A}}\right)^{-1}$ exists with a probability approaching 1 if the estimator is consistent. If one deals with the strict consistence, then this matrix exists only if the random size of the sample is sufficiently large. In principle, this size could be arbitrarily large and thus one faces a problem trying to modify the estimator in such a way that the asymptotic properties remain the same and that the inverse matrix exists with probability 1 even if the number of observations is comparatively small. This estimator allows one to get a more accurate estimation when evaluating $\left(A^{T} A-V_{\tilde{A}}\right)^{\dagger}$, since the probability of random deviations equals zero in this case.

The modification mentioned above is constructed as follows. Let

$$
T=\left[\begin{array}{cc}
B^{T} B & B^{T} A \\
A^{T} B & A^{T} A
\end{array}\right]=\left[\begin{array}{ll}
B & A
\end{array}\right]^{T} \cdot\left[\begin{array}{ll}
B & A
\end{array}\right], \quad W=\left[\begin{array}{cc}
0 & 0 \\
0 & V_{\tilde{A}}
\end{array}\right]
$$

be $(n+d) \times(n+d)$ matrices. 
Let $\lambda_{A}$ be the least positive root of the equation $\operatorname{det}(T-\lambda W)=0$ (if it exists at all). Put

$$
\mu= \begin{cases}\frac{m-\alpha}{m}, & \lambda_{A}>1+\frac{1}{m}, \\ \lambda_{A} \cdot \frac{m-\alpha}{m+1}, & \lambda_{A} \leqslant 1+\frac{1}{m},\end{cases}
$$

where $\alpha$ is chosen in the interval $(0, m)$. Following the suggestion in [2], one can choose $\alpha=n d+1$ if the number of observations is such that $m>n d+1$. Then the adjusted estimator is defined by

$$
\hat{X}_{\text {MALS }} \stackrel{\text { def }}{=}\left(A^{T} A-\mu V_{\tilde{A}}\right)^{\dagger} A^{T} B .
$$

In what follows we need yet another assumption.

(xiv) : The distributions $L(\tilde{a}(j)), 1 \leqslant j \leqslant n$, and $L(\tilde{b}(l)), 1 \leqslant l \leqslant d$, do not have atoms, that is, $\mathrm{P}(\tilde{a}(j)=z)=0$ for all $j=1, \ldots, n$ and $z \in \mathbb{R}$; similar property is assumed to hold for $\tilde{b}(l), 1 \leqslant l \leqslant d$.

Assumption (xiv allows one to prove the following auxiliary result.

Lemma 4.1. Let assumptions (ii), (iii), and (xiv) hold. Then $T>0$ almost surely for all $m \geqslant n+d$.

Proof. We show that

$$
\forall m \geqslant n+d: \quad \operatorname{rank}\left[\begin{array}{ll}
B & A
\end{array}\right]=n+d \quad \text { almost surely. }
$$

It follows from (8) that

$$
x^{T} T x=x^{T}\left[\begin{array}{ll}
B & A
\end{array}\right]^{T}\left[\begin{array}{ll}
B & A
\end{array}\right] x=\left\|\left[\begin{array}{ll}
B & A
\end{array}\right] x\right\|^{2} \geqslant 0
$$

for all $x \in \mathbb{R}^{n+d}$, whence $x^{T} T x=0 \Leftrightarrow\left[\begin{array}{ll}B & A\end{array}\right] x=0$. We have $\left[\begin{array}{ll}B & A\end{array}\right] \in \mathbb{R}^{m \times(n+d)}$. Since $\operatorname{rank}\left[\begin{array}{ll}B & A\end{array}\right]=n+d$ almost surely, we conclude that $\operatorname{Ker}\left[\begin{array}{ll}B & A\end{array}\right]=\left\{\begin{array}{l}0\} \\ \text { almost surely, }\end{array}\right.$ that is, $\left[\begin{array}{ll}B & A\end{array}\right] x=0 \Leftrightarrow x=0$. Thus $x^{T} T x>0$ almost surely for all $x \in \mathbb{R}^{n+d} \backslash\{0\}$, that is, $T>0$ almost surely.

To prove property (8) note that assumptions (ii) and (iii) imply that the vectors $\left\{\left(\begin{array}{ll}b_{i}^{T} & a_{i}^{T}\end{array}\right)^{T}, 1 \leqslant i \leqslant n+d\right\}$ are jointly independent. Applying condition xiv we prove that

$$
\mathrm{P}\left(\alpha_{1}\left(\begin{array}{ll}
b_{1}^{T} & a_{1}^{T}
\end{array}\right)^{T}+\cdots+\alpha_{n+d}\left(\begin{array}{ll}
b_{n+d}^{T} & a_{n+d}^{T}
\end{array}\right)^{T}=0\right)=0
$$

for an arbitrary family of numbers $\alpha_{1}, \ldots, \alpha_{n+d}$ belonging to $\mathbb{R}$. Hence

$$
\left\{\left(\begin{array}{ll}
b_{i}^{T} & a_{i}^{T}
\end{array}\right)^{T}, 1 \leqslant i \leqslant n+d\right\}
$$

is a linearly independent system almost surely and

$$
\operatorname{rank}\left[\begin{array}{cc}
b_{1}^{T} & a_{1}^{T} \\
b_{2}^{T} & a_{2}^{T} \\
\ldots & \ldots \\
b_{n+d}^{T} & a_{n+d}^{T}
\end{array}\right]=n+d=\operatorname{rank}\left[\begin{array}{cc}
b_{1}^{T} & a_{1}^{T} \\
b_{2}^{T} & a_{2}^{T} \\
\ldots & \ldots \\
b_{m}^{T} & a_{m}^{T}
\end{array}\right]
$$

for all $m \geqslant n+d$.

Lemma 4.2. Let assumptions (1i), (iii), and (xiv) hold. Then, for all $m \geqslant n+d$,

1) $\mu$ and $X_{\text {MALS }}$ are well defined almost surely;

2) $A^{T} A-\mu V_{\tilde{A}} \geqslant \frac{\alpha+1}{m+1} A^{T} A>0$ almost surely. 
Proof. All the inequalities in the proof below hold with probability one.

1) We have $T-\lambda W=T^{-1 / 2}\left(I_{n+d}-\lambda T^{-1 / 2} W T^{-1 / 2}\right) T^{-1 / 2}$. Thus

$$
\begin{aligned}
\operatorname{det}(T-\lambda W)=0 & \Leftrightarrow \operatorname{det}\left(I_{n+d}-\lambda T^{-1 / 2} W T^{-1 / 2}\right)=0 \\
& \Leftrightarrow \operatorname{det}\left(T^{-1 / 2} W T^{-1 / 2}-\frac{1}{\lambda} I_{n+d}\right)=0 .
\end{aligned}
$$

Since $T^{-1 / 2} W T^{-1 / 2} \geqslant 0$, we conclude that $\lambda_{A}=\left(\lambda_{\max }\left(T^{-1 / 2} W T^{-1 / 2}\right)\right)^{-1}$ exists almost surely. Hence $\mu$ and $X_{\text {MALS }}$ are well defined.

2) Next, $\mu \leqslant \lambda_{A} \frac{m-\alpha}{m+1}$, whence

$$
A^{T} A-\mu V_{\tilde{A}} \geqslant A^{T} A-\lambda_{A} \frac{m-\alpha}{m+1} V_{\tilde{A}}=\frac{m-\alpha}{m+1}\left(A^{T} A-\lambda_{A} V_{\tilde{A}}\right)+\frac{\alpha+1}{m+1} A^{T} A .
$$

Consider

$$
T-\lambda_{A} W=T^{-1 / 2}\left(I_{n+d}-\lambda_{A} T^{-1 / 2} W T^{-1 / 2}\right) T^{-1 / 2} .
$$

Since $\lambda_{A}=\lambda_{\max }^{-1}\left(T^{-1 / 2} W T^{-1 / 2}\right)$, we obtain

$$
\lambda_{A} T^{-1 / 2} W T^{-1 / 2} \leqslant I_{n+d},
$$

whence $T-\lambda_{A} W \geqslant 0$. Further,

$$
T-\lambda_{A} W=\left[\begin{array}{cc}
B^{T} B & B^{T} A \\
A^{T} B & A^{T} A-\lambda_{A} V_{\tilde{A}}
\end{array}\right] \geqslant 0 \Rightarrow A^{T} A-\lambda_{A} V_{\tilde{A}} \geqslant 0 .
$$

Since

$$
T=\left[\begin{array}{ll}
B^{T} B & B^{T} A \\
A^{T} B & A^{T} A
\end{array}\right]>0,
$$

the matrix $A^{T} A$ is positive definite, that is, $A^{T} A>0$.

Using expansion (9), we establish $A^{T} A-\lambda_{A} V_{\tilde{A}} \geqslant \frac{\alpha+1}{m+1} A^{T} A>0$.

Therefore,

$$
\hat{X}_{\mathrm{MALS}}=\left(A^{T} A-\mu V_{\tilde{A}}\right)^{-1} \times A^{T} B
$$

exists with probability one for all $m \geqslant n+d$, which is comparable with the sizes of the matrices. In addition, the estimator is evaluated with the help of inversion (which is different from the pseudo inversion) of some positive definite matrix. This feature leads to better numerical properties of the adjusted estimator.

Yet another assumption is needed to prove that the asymptotic properties of the adjusted estimator remain the same.

$$
(\mathbf{x v}): V_{\tilde{b}}=\operatorname{Cov}(\tilde{b})>0 .
$$

Lemma 4.3. Let assumptions (ii)-(iii), (vii), (viii), (xiv), and (XV) hold. Then

$$
\sqrt{m}(1-\mu) \stackrel{\mathrm{P}}{\rightarrow} 0, \quad m \rightarrow \infty .
$$

The proof of Lemma 4.3 is given in the Appendix.

Lemma 4.3 is helpful in the proof of the theorem about the asymptotic properties of the adjusted estimator.

Theorem 4.1. Let assumptions (ii)-(iii), (vii), (viii), (Xiv), and (区V) hold. Then

$$
\sqrt{m}\left(\hat{X}_{\mathrm{MALS}}-\hat{X}_{\mathrm{ALS}}\right) \stackrel{\mathrm{P}}{\rightarrow} 0, \quad m \rightarrow \infty .
$$


Proof. The equalities

$$
\left(A^{T} A-\mu V_{\tilde{A}}\right) \hat{X}_{\mathrm{MALS}}=A^{T} B
$$

and

$$
\left(A^{T} A-V_{\tilde{A}}\right) \hat{X}_{\mathrm{ALS}}=A^{T} B
$$

yield

$$
\left(A^{T} A-\mu V_{\tilde{A}}\right) \hat{X}_{\mathrm{MALS}}-\left(A^{T} A-V_{\tilde{A}}\right) \hat{X}_{\mathrm{ALS}}=0 .
$$

Therefore,

$$
\left(A^{T} A-\mu V_{\tilde{A}}\right)\left(\hat{X}_{\mathrm{MALS}}-\hat{X}_{\mathrm{ALS}}\right)=-(1-\mu) V_{\tilde{A}} \hat{X}_{\mathrm{ALS}}
$$

Further,

$$
\begin{gathered}
V_{A_{0}}^{-1}\left(A^{T} A-\mu V_{\tilde{A}}\right) \sqrt{m}\left(\hat{X}_{\mathrm{MALS}}-\hat{X}_{\mathrm{ALS}}\right)=-\sqrt{m}(1-\mu) V_{A_{0}}^{-1} V_{\tilde{A}} \hat{X}_{\mathrm{ALS}} \\
V_{A_{0}}^{-1}\left(A^{T} A-\mu V_{\tilde{A}}\right)=V_{A_{0}}^{-1}\left(A^{T} A-V_{\tilde{A}}\right)+(1-\mu) V_{A_{0}}^{-1} V_{\tilde{A}} \\
=V_{A_{0}}^{-1}\left(A^{T} A-V_{\tilde{A}}\right)+(1-\mu)\left(\frac{1}{m} V_{A_{0}}\right)^{-1} \operatorname{Cov}(\tilde{a}) \\
\quad \underset{m \rightarrow \infty}{\stackrel{\mathrm{P}}{m}} I_{n}+0 \cdot V_{A_{\infty}}^{-1} \operatorname{Cov}(\tilde{a})=I_{n}-\sqrt{m}(1-\mu) V_{A_{0}}^{-1} V_{\tilde{A}} \hat{X}_{\mathrm{ALS}} \\
=-\sqrt{m}(1-\mu)\left(\frac{1}{m} V_{A_{0}}\right)^{-1} \operatorname{Cov}(\tilde{a}) \hat{X}_{\mathrm{ALS}} \\
\underset{\mathrm{P} \rightarrow \infty}{\mathrm{P}} 0 \cdot V_{A_{\infty}}^{-1} \operatorname{Cov}(\tilde{a}) X_{0}=0 .
\end{gathered}
$$

Taking into account equality (3), we get

$$
\sqrt{m}\left(\hat{X}_{\mathrm{MALS}}-\hat{X}_{\mathrm{ALS}}\right) \stackrel{\mathrm{P}}{\rightarrow} 0, \quad m \rightarrow \infty .
$$

\section{Concluding Remarks}

Sufficient conditions are obtained in this paper for the asymptotic normality of the adjusted least squares estimator. A consistent estimator of the corresponding asymptotic covariance matrix is given. An adjusted estimator is constructed with the help of an inverse matrix that exists with probability one even if the size of a sample is small. The adjusted estimator possesses the same asymptotic properties as the original estimator.

\section{ApPEndix}

6.1. Proof of convergence (5). Put

$$
\begin{aligned}
\eta_{i} & =\frac{1}{\sqrt{m}} \operatorname{vec}\left(a_{i} \tilde{b}_{i}^{T}+\mathrm{E} \tilde{a}_{i} \tilde{a}_{i}^{T} X_{0}-a_{i} \tilde{a}_{i}^{T} X_{0}\right) \\
& =\frac{1}{\sqrt{m}} \operatorname{vec}\left(a_{i} \tilde{b}_{i}^{T}-a_{i}^{0} \tilde{a}_{i}^{T} X_{0}-\left(\tilde{a}_{i} \tilde{a}_{i}^{T}-\mathrm{E} \tilde{a}_{i} \tilde{a}_{i}^{T}\right) X_{0}\right) .
\end{aligned}
$$

Then

$$
\frac{l}{\sqrt{m}}=\sum_{i=1}^{m} \eta_{i}, \quad \mathrm{E} \eta_{i}=0 .
$$

Now we check the assumptions of the Lyapunov central limit theorem for random vectors (see Theorem 19 in [8, Section IV.4]). First we show that the limit

$$
\lim _{m \rightarrow \infty} \sum_{i=1}^{m} \operatorname{Cov}\left(\eta_{i}\right)
$$


exists. Denote $\xi_{1}=\xi_{1}(i)=\operatorname{vec}\left(a_{i} \tilde{b}_{i}^{T}\right), \xi_{2}=\xi_{2}(i)=\operatorname{vec}\left(a_{i}^{0} \tilde{a}_{i}^{T} X_{0}\right)$, and

$$
\xi_{3}=\xi_{3}(i)=\operatorname{vec}\left(\left(\tilde{a}_{i} \tilde{a}_{i}^{T}-\mathrm{E} \tilde{a}_{i} \tilde{a}_{i}^{T}\right) X_{0}\right) .
$$

Then $\eta_{i}=\frac{1}{\sqrt{m}}\left(\xi_{1}-\xi_{2}-\xi_{3}\right)$ and

$$
\begin{aligned}
\operatorname{Cov}\left(\eta_{i}\right) & =\frac{1}{m} \mathrm{E}\left(\xi_{1}-\xi_{2}-\xi_{3}\right)\left(\xi_{1}-\xi_{2}-\xi_{3}\right)^{T} \\
& =\frac{1}{m}\left(\mathrm{E} \xi_{1} \xi_{1}^{T}+\mathrm{E} \xi_{2} \xi_{2}^{T}+\mathrm{E} \xi_{3} \xi_{3}^{T}+\mathrm{E} \xi_{2} \xi_{3}^{T}+\mathrm{E} \xi_{3} \xi_{2}^{T}\right) \\
& =\mathrm{E} \xi_{1} \xi_{1}^{T}+\mathrm{E}\left(\xi_{2}+\xi_{3}\right)\left(\xi_{2}+\xi_{3}\right)^{T} .
\end{aligned}
$$

Equality (1) implies that $\mathrm{E} \xi_{1} \xi_{2}^{T}=\mathrm{E} \xi_{2} \xi_{1}^{T}=\mathrm{E} \xi_{1} \xi_{3}^{T}=\mathrm{E} \xi_{3} \xi_{1}^{T}=0$.

The terms in equality (10) are estimated separately by using the properties of the Kronecker product [5, Section 12]. For the first term we have

$$
\begin{aligned}
\mathrm{E} \xi_{1} \xi_{1}^{T} & =\mathrm{E} \operatorname{vec}\left(a_{i} \tilde{b}_{i}^{T}\right) \operatorname{vec}^{T}\left(a_{i} \tilde{b}_{i}^{T}\right)=\mathrm{E}\left(\tilde{b}_{i} \otimes a_{i}\right)\left(\tilde{b}_{i}^{T} \otimes a_{i}^{T}\right)=\mathrm{E}\left(\tilde{b}_{i} \tilde{b}_{i}^{T} \otimes a_{i} a_{i}^{T}\right) \\
& =\mathrm{E}\left(\tilde{b}_{i} \tilde{b}_{i}^{T} \otimes a_{i}^{0} a_{i}^{0 T}+\tilde{b}_{i} \tilde{b}_{i}^{T} \otimes \tilde{a}_{i} \tilde{a}_{i}^{T}\right)=V_{\tilde{b}} \otimes a_{i}^{0} a_{i}^{0 T}+V_{\tilde{b}} \otimes V_{\tilde{a}} .
\end{aligned}
$$

The second term is such that

$$
\begin{aligned}
\mathrm{E} \xi_{2} \xi_{2}^{T} & =\mathrm{E} \operatorname{vec}\left(a_{i}^{0} \tilde{a}_{i}^{T} X_{0}\right) \operatorname{vec}^{T}\left(a_{i}^{0} \tilde{a}_{i}^{T} X_{0}\right)=\mathrm{E}\left(\left(X_{0}^{T} \tilde{a}_{i}\right) \otimes a_{i}^{0}\right)\left(\left(X_{0}^{T} \tilde{a}_{i}\right) \otimes a_{i}^{0}\right)^{T} \\
& =\mathrm{E}\left(\left(X_{0}^{T} \tilde{a}_{i}\right) \otimes\left(I_{n} a_{i}^{0}\right)\right)\left(\left(X_{0}^{T} \tilde{a}_{i}\right) \otimes\left(I_{n} a_{i}^{0}\right)\right)^{T} \\
& =\mathrm{E}\left(\left(X_{0}^{T} \otimes I_{n}\right)\left(\tilde{a}_{i} \otimes a_{i}^{0}\right)\right)\left(\left(X_{0}^{T} \otimes I_{n}\right)\left(\tilde{a}_{i} \otimes a_{i}^{0}\right)\right)^{T} \\
& =\left(X_{0}^{T} \otimes I_{n}\right) \mathrm{E}\left[\left(\tilde{a}_{i} \tilde{a}_{i}^{T}\right) \otimes\left(a_{i}^{0} a_{i}^{0 T}\right)\right]\left(X_{0}^{T} \otimes I_{n}\right)^{T}
\end{aligned}
$$

The third term is evaluated as follows

$$
\begin{aligned}
\mathrm{E} \xi_{3} \xi_{3}^{T}= & \mathrm{E} \operatorname{vec}\left(\left(\tilde{a}_{i} \tilde{a}_{i}^{T}-\mathrm{E} \tilde{a}_{i} \tilde{a}_{i}^{T}\right) X_{0}\right) \operatorname{vec}^{T}\left(\left(\tilde{a}_{i} \tilde{a}_{i}^{T}-\mathrm{E} \tilde{a}_{i} \tilde{a}_{i}^{T}\right) X_{0}\right) \\
= & \mathrm{E}\left(\left(X_{0}^{T} \tilde{a}_{i}\right) \otimes \tilde{a}_{i}-\mathrm{E}\left(X_{0}^{T} \tilde{a}_{i}\right) \otimes \tilde{a}_{i}\right)\left(\left(X_{0}^{T} \tilde{a}_{i}\right)^{T} \otimes \tilde{a}_{i}^{T}-\mathrm{E}\left(X_{0}^{T} \tilde{a}_{i}\right)^{T} \otimes \tilde{a}_{i}^{T}\right) \\
= & \mathrm{E}\left[\left(\left(X_{0}^{T} \tilde{a}_{i}\right) \otimes\left(I_{n} \tilde{a}_{i}\right)\right)\left(\left(X_{0}^{T} \tilde{a}_{i}\right)^{T} \otimes\left(I_{n} \tilde{a}_{i}\right)^{T}\right)\right] \\
& -\left[\mathrm{E}\left(X_{0}^{T} \tilde{a}_{i}\right) \otimes\left(I_{n} \tilde{a}_{i}\right)\right]\left[\mathrm{E}\left(X_{0}^{T} \tilde{a}_{i}\right)^{T} \otimes\left(I_{n} \tilde{a}_{i}\right)^{T}\right] \\
= & \mathrm{E}\left[\left(X_{0}^{T} \otimes I_{n}\right)\left(\tilde{a}_{i} \otimes \tilde{a}_{i}\right)\right]\left[\left(X_{0}^{T} \otimes I_{n}\right)\left(\tilde{a}_{i} \otimes \tilde{a}_{i}\right)\right]^{T} \\
& -\mathrm{E}\left[\left(X_{0}^{T} \otimes I_{n}\right)\left(\tilde{a}_{i} \otimes \tilde{a}_{i}\right)\right] \mathrm{E}\left[\left(X_{0}^{T} \otimes I_{n}\right)\left(\tilde{a}_{i} \otimes \tilde{a}_{i}\right)\right]^{T} \\
= & \left(X_{0}^{T} \otimes I_{n}\right)\left[\mathrm{E}\left(\tilde{a}_{i} \otimes \tilde{a}_{i}\right)\left(\tilde{a}_{i} \otimes \tilde{a}_{i}\right)-\operatorname{vec}\left(V_{\tilde{a}}\right) \operatorname{vec}^{T}\left(V_{\tilde{a}}\right)\right]\left(X_{0}^{T} \otimes I_{n}\right)^{T} .
\end{aligned}
$$

Next, for the fourth term,

$$
\begin{aligned}
\mathrm{E} \xi_{2} \xi_{3}^{T}= & \mathrm{E} \operatorname{vec}\left(a_{i}^{0} \tilde{a}_{i}^{T} X_{0}\right) \operatorname{vec}^{T}\left(\left(\tilde{a}_{i} \tilde{a}_{i}^{T}-\mathrm{E} \tilde{a}_{i} \tilde{a}_{i}^{T}\right) X_{0}\right) \\
= & \mathrm{E}\left(\left(X_{0}^{T} \tilde{a}_{i}\right) \otimes a_{i}^{0}\right)\left(\left(X_{0}^{T} \tilde{a}_{i}\right)^{T} \otimes \tilde{a}_{i}^{T}-\mathrm{E}\left(X_{0}^{T} \tilde{a}_{i}\right)^{T} \otimes \tilde{a}_{i}^{T}\right) \\
= & \mathrm{E}\left[\left(\left(X_{0}^{T} \tilde{a}_{i}\right) \otimes\left(I_{n} a_{i}^{0}\right)\right)\left(\left(X_{0}^{T} \tilde{a}_{i}\right)^{T} \otimes\left(I_{n} \tilde{a}_{i}\right)^{T}\right)\right] \\
& -\mathrm{E}\left[\left(X_{0}^{T} \tilde{a}_{i}\right) \otimes\left(I_{n} a_{i}^{0}\right)\right] \mathrm{E}\left[\left(X_{0}^{T} \tilde{a}_{i}\right)^{T} \otimes\left(I_{n} \tilde{a}_{i}\right)^{T}\right] \\
= & \mathrm{E}\left[\left(X_{0}^{T} \otimes I_{n}\right)\left(\tilde{a}_{i} \otimes a_{i}^{0}\right)\right]\left[\left(X_{0}^{T} \otimes I_{n}\right)\left(\tilde{a}_{i} \otimes \tilde{a}_{i}\right)\right]^{T} \\
= & \left(X_{0}^{T} \otimes I_{n}\right) \mathrm{E}\left[\left(\tilde{a}_{i} \otimes a_{i}^{0}\right)\left(\tilde{a}_{i} \otimes \tilde{a}_{i}\right)\right]\left(X_{0}^{T} \otimes I_{n}\right)^{T} .
\end{aligned}
$$


Finally, for the fifth term we have that

$$
\mathrm{E} \xi_{3} \xi_{2}^{T}=\left(X_{0}^{T} \otimes I_{n}\right) \mathrm{E}\left[\left(\tilde{a}_{i} \otimes a_{i}^{0}\right)\left(\tilde{a}_{i} \otimes \tilde{a}_{i}\right)\right]^{T}\left(X_{0}^{T} \otimes I_{n}\right)^{T}
$$

Recalling assumption (viii), we see that the first three terms do not depend on $i$. Thus we need to find the limit for the fourth term only (as a result, we find the limit for the fifth term, too):

$$
\begin{aligned}
\lim _{m \rightarrow \infty} \frac{1}{m} \sum_{i=1}^{m} \mathrm{E} \xi_{2} \xi_{3}^{T} & =\left(X_{0}^{T} \otimes I_{n}\right)\left[\lim _{m \rightarrow \infty} \sum_{i=1}^{m} \frac{1}{m} \mathrm{E}\left[\left(\tilde{a}_{i} \otimes a_{i}^{0}\right)\left(\tilde{a}_{i} \otimes \tilde{a}_{i}\right)\right]\right]\left(X_{0}^{T} \otimes I_{n}\right)^{T} \\
& =\left(X_{0}^{T} \otimes I_{n}\right)\left[\lim _{m \rightarrow \infty} \sum_{i=1}^{m} \frac{1}{m} \mathrm{E}\left[\left(\tilde{a} \otimes a_{i}^{0}\right)(\tilde{a} \otimes \tilde{a})\right]\right]\left(X_{0}^{T} \otimes I_{n}\right)^{T} \\
& =\left(X_{0}^{T} \otimes I_{n}\right) \mathrm{E}\left[\left(\tilde{a} \otimes\left[\lim _{m \rightarrow \infty} \frac{1}{m} \sum_{i=1}^{m} a_{i}^{0}\right]\right)(\tilde{a} \otimes \tilde{a})\right]\left(X_{0}^{T} \otimes I_{n}\right)^{T} \\
& =\left(X_{0}^{T} \otimes I_{n}\right) \mathrm{E}\left[\left(\tilde{a} \otimes a^{0}\right)(\tilde{a} \otimes \tilde{a})\right]\left(X_{0}^{T} \otimes I_{n}\right)^{T} .
\end{aligned}
$$

Now we consider the sum

$$
\begin{aligned}
\sum_{i=1}^{m} \operatorname{Cov}\left(\eta_{i}\right)=\frac{1}{m} \sum_{i=1}^{m}( & V_{\tilde{b}} \otimes\left(a_{i}^{0} a_{i}^{0 T}+V_{\tilde{a}}\right) \\
+ & \left(X_{0}^{T} \otimes I_{n}\right) \mathrm{E}\left(\tilde{a}_{i} \tilde{a}_{i}^{T}\right)\left(a_{i}^{0} a_{i}^{0 T}\right)\left(X_{0}^{T} \otimes I_{n}\right)^{T} \\
+ & \left(X_{0}^{T} \otimes I_{n}\right)\left[\mathrm{E}\left(\tilde{a}_{i} \otimes \tilde{a}_{i}\right)\left(\tilde{a}_{i} \otimes \tilde{a}_{i}\right)-\operatorname{vec}\left(V_{\tilde{a}}\right) \operatorname{vec}^{T}\left(V_{\tilde{a}}\right)\right] \\
& \times\left(X_{0}^{T} \otimes I_{n}\right)^{T} \\
+ & \left(X_{0}^{T} \otimes I_{n}\right) \mathrm{E}\left[\left(\tilde{a}_{i} \otimes a_{i}^{0}\right)\left(\tilde{a}_{i} \otimes \tilde{a}_{i}\right)\right]\left(X_{0}^{T} \otimes I_{n}\right)^{T} \\
& \left.+\left(X_{0}^{T} \otimes I_{n}\right) \mathrm{E}\left[\left(\tilde{a}_{i} \otimes a_{i}^{0}\right)\left(\tilde{a}_{i} \otimes \tilde{a}_{i}\right)\right]^{T}\left(X_{0}^{T} \otimes I_{n}\right)^{T}\right) \\
\longrightarrow V_{\tilde{b}} \otimes & V_{A_{\infty}}+V_{\tilde{b}} \otimes V_{\tilde{a}} \\
+ & \left(X_{0}^{T} \otimes I_{n}\right) \\
& \times\left[V_{\tilde{a}} \otimes V_{A_{\infty}}+\mathrm{E}(\tilde{a} \otimes \tilde{a})(\tilde{a} \otimes \tilde{a})-\operatorname{vec}\left(V_{\tilde{a}}\right) \operatorname{vec}\left(V_{\tilde{a}}\right)\right. \\
& \left.+\mathrm{E}\left[\left(\tilde{a} \otimes a^{0}\right)(\tilde{a} \otimes \tilde{a})\right]+\mathrm{E}\left[\left(\tilde{a} \otimes a^{0}\right)(\tilde{a} \otimes \tilde{a})\right]^{T}\right] \\
& \times\left(X_{0}^{T} \otimes I_{n}\right)^{T} \\
\stackrel{\text { def }}{=} \Sigma_{1} . &
\end{aligned}
$$

In particular, $\left\|\operatorname{Cov}\left(\eta_{m}\right)\right\| \rightarrow 0$ as $m \rightarrow \infty$ and thus

$$
x^{T} \operatorname{Cov}\left(\eta_{m}\right) x \rightarrow 0, \quad m \rightarrow \infty
$$

for all $x \in \mathbb{R}^{n d}$. Then we prove that the Lyapunov condition

$$
\sum_{i=1}^{m} \mathrm{E}\left\|\eta_{i}\right\|^{2+\nu} \rightarrow 0, \quad m \rightarrow \infty
$$


holds for some $\nu>0$. For this, we estimate the sum

$$
\begin{aligned}
\sum_{i=1}^{m}\left\|\mathrm{E} \eta_{i}\right\|^{2+\nu} & =\frac{1}{m^{1+\nu / 2}} \sum_{i=1}^{m} \mathrm{E}\left\|\xi_{1}-\xi_{2}-\xi_{3}\right\|^{2+\nu} \\
& \leqslant \frac{3^{2+\nu}}{m^{1+\nu / 2}} \sum_{i=1}^{m} \mathrm{E}\left(\left\|\xi_{1}\right\|^{2+\nu}+\left\|\xi_{2}\right\|^{2+\nu}+\left\|\xi_{3}\right\|^{2+\nu}\right) .
\end{aligned}
$$

Next, using conditions (viii), (ix), and ( $(\underline{\mathrm{x}})$ we estimate the terms separately for

$$
0<\nu \leqslant \max (\delta, \tau) \text {. }
$$

For the first term, we have

$$
\begin{gathered}
\frac{1}{m^{1+\nu / 2}} \sum_{i=1}^{m} \mathrm{E}\left\|\xi_{1}\right\|^{2+\nu} \\
=\frac{1}{m^{1+\nu / 2}} \sum_{i=1}^{m} \mathrm{E}\left\|\tilde{b}_{i} \otimes a_{i}\right\|^{2+\nu} \\
\leqslant \frac{2^{2+\nu}}{m^{1+\nu / 2}} \sum_{i=1}^{m} \mathrm{E}\left(\left\|\tilde{b}_{i} \otimes a_{i}^{0}\right\|^{2+\nu}+\left\|\tilde{b}_{i} \otimes \tilde{a}_{i}\right\|^{2+\nu}\right) \\
\left\|\tilde{b}_{i} \otimes a_{i}^{0}\right\|^{2+\nu}=\left(\sum_{j=1}^{n} \sum_{k=1}^{d}\left(a_{i j}^{0} \tilde{b}_{i k}\right)^{2}\right)^{1+\nu / 2}=\left(\left\|a_{i}^{0}\right\|^{2} \cdot\left\|\tilde{b}_{i}\right\|^{2}\right)^{1+\nu / 2}=\left\|a_{i}^{0}\right\|^{2+\nu} \cdot\left\|\tilde{b}_{i}\right\|^{2+\nu} \\
\left\|\tilde{b}_{i} \otimes \tilde{a}_{i}\right\|^{2+\nu}=\left\|\tilde{a}_{i}\right\|^{2+\nu} \cdot\left\|\tilde{b}_{i}\right\|^{2+\nu} .
\end{gathered}
$$

Then

$$
\begin{aligned}
\frac{1}{m^{1+\nu / 2}} \sum_{i=1}^{m} \mathrm{E}\left\|\xi_{1}\right\|^{2+\nu} & =\frac{2^{2+\nu}}{m^{1+\nu / 2}} \sum_{i=1}^{m} \mathrm{E}\left(\left\|a_{i}^{0}\right\|^{2+\nu} \cdot \mathrm{E}\|\tilde{b}\|^{2+\nu}+\mathrm{E}\|\tilde{a}\|^{2+\nu} \cdot \mathrm{E}\|\tilde{b}\|^{2+\nu}\right) \\
& \leqslant \frac{\text { const }}{m^{\nu / 2}} \rightarrow 0, \quad m \rightarrow \infty .
\end{aligned}
$$

The second term is estimated by

$$
\begin{aligned}
\frac{1}{m^{1+\nu / 2}} \sum_{i=1}^{m} \mathrm{E}\left\|\xi_{2}\right\|^{2+\nu} & =\frac{1}{m^{1+\nu / 2}} \sum_{i=1}^{m}\left\|\left(X_{0}^{T} a_{i}^{0}\right) \otimes \tilde{a}_{i}\right\|^{2+\nu} \\
& =\frac{1}{m^{1+\nu / 2}} \sum_{i=1}^{m}\left\|X_{0}^{T} a_{i}^{0}\right\|^{2+\nu} \mathrm{E}\left\|\tilde{a}_{i}\right\|^{2+\nu} \leqslant \frac{\text { const }}{m^{\nu / 2}} \rightarrow 0, \quad m \rightarrow \infty .
\end{aligned}
$$

The third term is such that

$$
\begin{aligned}
\frac{1}{m^{1+\nu / 2}} \sum_{i=1}^{m} \mathrm{E}\left\|\xi_{3}\right\|^{2+\nu} & =\frac{1}{m^{1+\nu / 2}} \sum_{i=1}^{m} \mathrm{E}\left\|\left(X_{0}^{T} \tilde{a}_{i}\right) \otimes \tilde{a}_{i}-\mathrm{E}\left(X_{0}^{T} \tilde{a}_{i}\right) \otimes \tilde{a}_{i}\right\|^{2+\nu} \\
& =\frac{1}{m^{\nu / 2}} \mathrm{E}\left\|\left(X_{0}^{T} \tilde{a}\right) \otimes \tilde{a}-\mathrm{E}\left(X_{0}^{T} \tilde{a}\right) \otimes \tilde{a}\right\|^{2+\nu} \rightarrow 0, \quad m \rightarrow \infty .
\end{aligned}
$$

Thus the convergence in (12) holds and assumptions for the central limit theorem hold. Therefore

$$
\frac{l}{\sqrt{m}} \stackrel{d}{\rightarrow} N\left(0, \Sigma_{1}\right)
$$

6.2. Proof of statement 2 in Theorem 3.1. It follows from (vii) and (xii) that

$$
V_{\tilde{b}} \otimes V_{A_{\infty}}+V_{\tilde{b}} \otimes V_{\tilde{a}}>0,
$$

whence $\Sigma_{1}>0$ by (10). Then (vii) together with (6) implies that $\Sigma>0$. 
6.3. Proof of Lemma 4.3, Consider the difference

$$
T-W=\left[\begin{array}{cc}
B^{T} B & B^{T} A \\
A^{T} B & A^{T} A-V_{\tilde{A}}
\end{array}\right] .
$$

We derive from (ii)-(iii) and (viii) that

$$
\begin{gathered}
m^{-1}\left(A^{T} A-V_{\tilde{A}}\right) \underset{m \rightarrow \infty}{\stackrel{\mathrm{P}}{\longrightarrow}} V_{A_{\infty}}, \quad m^{-1} A^{T} B \underset{m \rightarrow \infty}{\stackrel{\mathrm{P}}{\longrightarrow}} V_{A_{\infty}} X_{0}, \\
m^{-1} B^{T} A \underset{m \rightarrow \infty}{\stackrel{\mathrm{P}}{\longrightarrow}} X_{0} V_{A_{\infty}} .
\end{gathered}
$$

Then we consider the term $m^{-1} B^{T} B$ :

$$
\begin{gathered}
\frac{1}{m} B^{T} B=\frac{1}{m} \sum_{i=1}^{m}\left(b_{i}^{0} b_{i}^{0 T}+b_{i}^{0} \tilde{b}_{i}^{T}+\tilde{b}_{i} b_{i}^{0 T}+\tilde{b}_{i} \tilde{b}_{i}^{T}\right), \\
\frac{1}{m} \sum_{i=1}^{m} b_{i}^{0} b_{i}^{0 T}=\frac{1}{m} B_{0}^{T} B_{0}=\frac{1}{m} A_{0} X_{0} X_{0}^{T} A_{0}^{T} \rightarrow X_{0} V_{A_{\infty}} X_{0}^{T}, \quad m \rightarrow \infty .
\end{gathered}
$$

The latter convergence follows from assumption (vii).

Now (iii), (viii), and the strong law of large numbers (Theorem 3 in [9, Section IV.3]) imply that

$$
m^{-1} \sum_{i=1}^{m} \tilde{b}_{i} \tilde{b}_{i}^{T} \stackrel{\mathrm{P} 1}{\longrightarrow} \operatorname{Cov}(\tilde{b})=V_{\tilde{b}}, \quad m \rightarrow \infty .
$$

Then we prove that

$$
m^{-1} \sum_{i=1}^{m} b_{i}^{0} \tilde{b}_{i} \stackrel{\mathrm{P}}{\rightarrow} 0 \quad \text { and } \quad m^{-1} \sum_{i=1}^{m} \tilde{b}_{i} b_{i}^{0} \stackrel{\mathrm{P}}{\rightarrow} 0
$$

as $m \rightarrow \infty$. We apply the Chebyshev law of large numbers to $m^{-1} \sum_{i=1}^{m} b_{i}^{0} \tilde{b}_{i}$. Note that $b_{k}^{0} \tilde{b}_{k}^{T}=\left(b_{k l}^{0} \tilde{b}_{k l}\right)_{i, j=1}^{d}, \quad \mathrm{E} b_{k l}^{0} \tilde{b}_{k j}=0, \quad \operatorname{Var} b_{k l}^{0} \tilde{b}_{k j}=0, \quad \operatorname{Var} \tilde{b}_{k j} \leqslant\left(b_{k l}^{0}\right)^{2} \sup _{k, j} \mathrm{E} \tilde{b}_{k l}^{2}$ for all $k \geqslant 1$. Completing the proof, one needs to show that

$$
\frac{\left(b_{k l}^{0}\right)^{2}}{k} \rightarrow 0, \quad k \rightarrow \infty
$$

for all $i=1, \ldots, d$ or that

$$
\frac{\left\|b_{k}^{0}\right\|^{2}}{k} \rightarrow \infty, \quad k \rightarrow \infty
$$

which is the same.

Indeed,

$$
\left\|b_{k}^{0}\right\|^{2}=\left\|X_{0}^{T} a_{k}^{0}\right\|^{2} \leqslant\left\|X_{0}\right\|_{\mathrm{F}}^{2}\left\|a_{k}^{0}\right\|^{2} .
$$

Denote $A_{0, m}=\left[\begin{array}{lll}a_{1}^{0 T} & \ldots & a_{m}^{0 T}\end{array}\right]$. Then

$$
\frac{1}{m}\left\|a_{m}^{0}\right\|^{2}=\frac{1}{m}\left(\operatorname{tr} A_{0, m}^{T} A_{0, m}-\operatorname{tr} A_{0, m-1}^{T} A_{0, m-1}\right) \underset{m \rightarrow \infty}{\longrightarrow} \operatorname{tr} V_{A_{\infty}}-\operatorname{tr} V_{A_{\infty}}=0 .
$$

The Chebyshev law of large numbers implies that

$$
\frac{1}{m} \sum_{i=1}^{m} b_{i}^{0} \tilde{b}_{i} \stackrel{\mathrm{P}}{\rightarrow} 0, \quad m \rightarrow \infty,
$$


whence we obtain $m^{-1} \sum_{i=1}^{m} \tilde{b}_{i} b_{i}^{0} \stackrel{\mathrm{P}}{\rightarrow} 0$ as $m \rightarrow \infty$. Finally,

$$
\begin{gathered}
\frac{1}{m}(T-W)=\frac{1}{m}\left[\begin{array}{cc}
B^{T} B & B^{T} A \\
A^{T} B & A^{T} A-V_{\tilde{A}}
\end{array}\right] \\
\underset{m \rightarrow \infty}{\stackrel{\mathrm{P}}{\longrightarrow}}\left[\begin{array}{cc}
X_{0}^{T} V_{A_{\infty}} X_{0}+V_{\tilde{b}} & X_{0}^{T} V_{A_{\infty}} \\
V_{A_{\infty}} X_{0} & V_{A_{\infty}}
\end{array}\right]=\left[\begin{array}{ll}
X_{0} & I_{n}
\end{array}\right]^{T} V_{A_{\infty}}\left[\begin{array}{ll}
X_{0} & I_{n}
\end{array}\right]+\left[\begin{array}{cc}
V_{\tilde{b}} & 0 \\
0 & 0
\end{array}\right] \\
\stackrel{\text { def }}{=} S_{\infty} .
\end{gathered}
$$

Now we prove that $S_{\infty}>0$. Indeed,

$$
\begin{aligned}
x^{T} V_{A_{\infty}} x & =x^{T}\left[\begin{array}{ll}
X_{0} & I_{n}
\end{array}\right]^{T} V_{A_{\infty}}\left[\begin{array}{ll}
X_{0} & I_{n}
\end{array}\right] x+x^{T}\left[\begin{array}{cc}
V_{\tilde{b}} & 0 \\
0 & 0
\end{array}\right] x \\
& =\left\|V_{A_{\infty}}\left(X_{0} x_{1}+x_{2}\right)\right\|^{2}+x_{1}^{T} V_{\tilde{b}} x_{1} \geqslant 0
\end{aligned}
$$

for all $x \in \mathbb{R}^{n+d}, x=\left[\begin{array}{l}x_{1} \\ x_{2}\end{array}\right]$, where $x_{1} \in \mathbb{R}^{n}$ and $x_{2} \in \mathbb{R}^{d}$. Thus

$$
x^{T} S_{\infty} x=0 \Leftrightarrow\left\{\begin{array} { l } 
{ X _ { 0 } x _ { 1 } + x _ { 2 } = 0 , } \\
{ x _ { 1 } = 0 . }
\end{array} \Leftrightarrow \left\{\begin{array}{l}
x_{1}=0, \\
x_{2}=0 .
\end{array}\right.\right.
$$

Then

$$
x^{T} S_{\infty} x>0
$$

for all $x \in \mathbb{R}^{n+d} \backslash\{0\}$. Therefore,

$$
\begin{aligned}
\mathrm{P}\left(\frac{1}{m}(T-W)>0\right) \rightarrow 1, \quad m \rightarrow \infty, \\
\quad \Longleftrightarrow \mathrm{P}((T-W)>0) \rightarrow 1, \quad m \rightarrow \infty, \\
\Longleftrightarrow \mathrm{P}\left(\lambda_{A}>1\right) \rightarrow 1, \quad m \rightarrow \infty .
\end{aligned}
$$

Further,

$$
\begin{aligned}
\left\{\lambda_{A}>1\right\} \subset\{ & \left.\frac{m-\alpha}{m+1}<\mu<\frac{m-\alpha}{m}\right\} \\
= & \left\{\frac{\alpha}{m} \leqslant 1-\mu<\frac{1+\alpha}{m+1}\right\} \\
& =\left\{\frac{\alpha}{m} \leqslant \sqrt{m}(1-\mu)<(1+\alpha) \frac{\sqrt{m}}{m+1}\right\},
\end{aligned}
$$

whence we conclude that $\sqrt{m}(1-\mu) \stackrel{\mathrm{P}}{\rightarrow} 0$ as $m \rightarrow \infty$.

\section{BIBLIOGRAPHY}

1. R. J. Carrol, D. Ruppert, and L. A. Sefanski, Measurement Error in Nonlinear Models, Monographs on Statistics and Applied Probability, vol. 63, Chapman \& Hall/CRC, London, 1995. MR:1630517 (2000c:62001)

2. C.-L. Cheng, H. Schneeweiss, and M. Thamerus, A small sample estimator for a polynomial regression with errors in the variables, J. R. Statist. Soc. B 62 (2000), no. 4, 699-709. MR1796286

3. W. A. Fuller, Measurement Error Models, Wiley, New York, 1987. MR898653 (89a:62160)

4. P. P. Gallo, Consistency of regression estimates when some variables are subject to error, Comm. Statist. A-Theory Methods 11 (1982), 973-983. MR655466 (83h:62106)

5. R. Bellman, Introduction to Matrix Analysis, McGraw-Hill Book Company, Inc., New YorkToronto-London, 1960. MR0122820 (23:A153)

6. F. R. Gantmacher, The Theory of Matrices, "Nauka", Moscow, 1988; English transl., AMS Chelsea Publishing, Providence, Rhode Island, 2000. MR986246 (90a:15001)

7. I. O. Sen'ko, The consistence of an adjusted least squares estimator in a linear vector errorsin-variables model (to appear). 
8. V. V. Petrov, Limit Theorems of Probability Theory: Sequences of Independent Random Variables, "Nauka", Moscow, 1987; English transl., Clarendon Press, Oxford, 1995. MR1353441 (96h:60048)

9. A. N. Shiryaev, Probability, "Nauka", Moscow, 1980; English transl., Springer-Verlag, Berlin, 1994. MR609521(82d:60002)

Department of Mathematical Analysis, Faculty for Mechanics and Mathematics, National Taras Shevchenko University, Volodymyrs'Ka Street, 64, Kyiv 01601, Ukraine

E-mail address: ivan_senko@ukr.net

Received 25/OCT/2012

Translated by N. SEMENOV 